\title{
Principales retos educativos de los profesores de la Unidad Académica del Norte del Estado de Nayarit durante la pandemia de COVID-19
}

\section{Main educational challenges of the professors of the Academic Unit of the North of the State of Nayarit during the COVID-19 pandemic}

\author{
CHÁVEZ-SÁNCHEZ, Gabriela†**, HERNÁNDEZ-GARCÍA, Juvencio y GONZÁLEZ-BASILIO, \\ Sofía de Jesús
}

\author{
Universidad Autónoma de Nayarit, México. \\ ID $1^{\mathrm{er}}$ Autor: Gabriela, Chávez-Sánchez \\ ID $1^{\text {er }}$ Coautor: Juvencio, Hernández-García \\ ID $2^{\text {do }}$ Coautor: Sofía de Jesús, González-Basilio
}

DOI: $10.35429 / \mathrm{JHS} .2020 .12 .4 .20 .30$

Recibido Julio 20, 2020; Aceptado Diciembre 30, 2020

\section{Resumen}

Este articulo presenta las problemáticas y retos educativos que los docentes de la Unidad Académica del Norte del Estado de Nayarit (UAEN) han manifestado durante la crisis sanitaria que se vive actualmente derivada de la pandemia con la COVID-19, con el objetivo de documentar la experiencia y proponer recomendaciones que permitan repensar la práctica docente. Para su elaboración se utilizó un cuestionario adaptado del instrumento aplicado por la Universidad Nacional Autónoma de México (UNAM, 2020), el cual fue aplicado a docentes activos en la Unidad Académica a través de un formulario de google. En el cuál se recogen datos estadísticos del personal y las opiniones de los participantes, relacionadas con esta temática, lo cual permitirá diseñar una serie de acciones encaminadas a mejorar los procesos a los cuales se enfrentan las instituciones educativas no solo en educación superior sino en todos los tipos y niveles educativos, pero en especial de la Universidad Autónoma de Nayarit.

Docentes, retos educativos, educación en modalidad no escolar

\begin{abstract}
This article presents the educational problems and challenges that the teachers of the Academic Unit of the North of the State of Nayarit (UAEN) have manifested during the current health crisis derived from the pandemic with COVID-19, with the aim of documenting the experience and propose recommendations that rethink teaching practice. For its preparation, a questionnaire adapted from the instrument applied by the National Autonomous University of Mexico (UNAM, 2020) was used, which was applied to active teachers in the Academic Unit through a google form. In which statistical data of the personnel and the opinions of the participants are collected, related to this issue, which will allow the design of a series of actions aimed at improving the processes faced by educational institutions not only in higher education but in all the types and levels of education, but especially from the Autonomous University of Nayarit.
\end{abstract}

Teachers, educational challenges and non-school education

Citación: CHÁVEZ-SÁNCHEZ, Gabriela, HERNÁNDEZ-GARCÍA, Juvencio y GONZÁLEZ-BASILIO, Sofía de Jesús. Principales retos educativos de los profesores de la Unidad Académica del Norte del Estado de Nayarit durante la pandemia de COVID-19. Revista de Educación Superior. 2020. 4-12:20-30.

\footnotetext{
*Correspondencia al Autor (Correo Electrónico: gabriela.chavez@uan.edu.mx)

$\dagger$ Investigador contribuyendo como primer autor.
} 


\section{Introducción}

La suspensión de clases presenciales en el mes de marzo de 2020 en todos los tipos y niveles educativos, trajo como consecuencia grandes cambios en el proceso educativo que afectaron directamente a docentes, estudiantes y familias en general, "lo cual ha creado numerosos efectos en los actores del proceso educativo" (Sánchez et al, 2020). En el caso de las Universidades e Instituciones de Educación Superior no han sido la excepción, al tener que apegarse a los lineamientos que las autoridades gubernamentales han decretado con el fin de seguir desarrollando las diversas actividades de sus funciones sustantivas.

Este panorama provocó que la docencia universitaria en la modalidad escolarizada migrara a la no escolarizada, sustentada por diversas herramientas y tecnologías para llevar a cabo los procesos formativos y con el conocimiento que el docente pudiera tener para realizar estos cambios en su praxis, lo que ha hecho visible las necesidades y deficiencias que tienen las instituciones en cuestiones de infraestructura, capacitación y conectividad.

Los docentes abandonaron el salón de clases que ha sido por años su espacio, para de manera obligada transformarse en docentes que crean ambientes virtuales de aprendizaje, diseñan contenido multimedia e hipermedia, manejan de sistemas de gestión del aprendizaje y ademas de llevar a cabo su clase con estudiantes que provienen de diversas comunidades, condiciones y características sociales y pedagógicas, lo que ha complejizado su quehacer en los procesos de aprendizaje ademas de considerar las presiones propias derivadas del confinamiento y las implicaciones económicas, afectivas, emocionales y de salud, que muchos de ellos han visto afectadas.

Esta situación trajo como consecuencia que el estilo de vida de todos se modificará, la educación en los diferentes niveles educativos cambió totalmente tomando por sorpresa a toda la población en general, el hogar se convirtió en un espacio educativo donde se desarrollan las clases, se llevan a cabo las actividades administrativas, se irrumpieron horarios, los límites entre casa y trabajo se desdibujaron, todo lo anterior para lograr cumplir con los requerimientos y objetivos que la educación actual demanda.
Es por ello que, el articulo presenta los resultados de una investigación realizada en la UAEN perteneciente a la Universidad Autónoma de Nayarit (UAN), esta Unidad Académica se ubica en la región norte del estado de Nayarit en el municipio de Acaponeta y que cuenta con una población proveniente de diferentes comunidades, con condiciones $\mathrm{y}$ características muy particulares que ha complicado el desarrollo de las actividades, por la falta de conectividad y acceso a recursos tecnológicos. En este trabajo se identifican y analizan las problemáticas a las que se enfrentan los docentes desde el aspecto tecnológico, pedagogía y socioafectivo; así como de algunas propuestas que contribuyan a mejorar las estrategias institucionales en estos tiempos.

\section{Antecedentes}

En diciembre del 2019, en China surgió un brote de infección respiratoria aguda causada por un nuevo coronavirus, provocando una ola de incertidumbre en todo el mundo por el aumento tan repentino de casos diarios y muertes por todo el mundo. Motivo por el cual la Organización Mundial de la Salud (OMS) declaró pandemia mundial la enfermedad. Siendo un virus altamente contagioso obligó a modificar nuestra forma de vida.

Incluso ya no se habla del término pandemia, sino que nos enfrentamos a lo que se ha denominado como sindemia, la cual ya no ubica la enfermedad de la COVID-19 como un problema exclusivo de salud sino que se ha extendido a las dimensiones sociales, económicas y educativas, lo que ha llevado a un replanteamiento de la vida tal como la conocíamos; en cada uno de los aspectos de nuestra "normalidad", se han tenido que gestar cambios que van desde un pequeño y casual apretón de manos hasta la convivencia entre 4 paredes.

En educación de tipo básico el reto es mayor y la complejidad se amplía por el número de estudiantes y contextos en los que se debe de trabajar, tal como lo comenta Martínez (2020), los retos históricos, hoy se ven potenciados con la pandemia y así es como lo hacen: 
"Como el resto de los mortales, con pasmo, frustración y miedo en algunos momentos. Con pasmo, porque la pandemia por el nuevo virus nos sorprendió a todos. Con frustración, cuando advertimos que, por años, subestimamos el uso de la tecnología en nuestra práctica docente y hoy debemos recurrir a ella para continuar con la enseñanza a distancia. Con miedo, porque transitar de una educación presencial a otra que plantea la implementación del uso de internet y de Tecnologías de la Información y la Comunicación en el proceso educativo no es nada sencillo, sobre todo si consideramos que ese tránsito se dio de forma súbita y sin la capacitación adecuada".

Desde luego, en este panorama se encuentra una sensación de desconcierto, decepción y enojo también en los académicos de la educación superior; desconcierto cuando de la noche a la mañana nuestro hogar se convirtió en salón de clases, sala de juntas, centro de capacitación virtual, oficina de atención psicopedagógica, y espacio para brindar asesoría y tutoría a distancia.

Decepción, cuando advertimos que seguimos confundiendo la escolarización con la educación, cuando nos damos cuenta que estamos obstinados en cubrir contenidos y no en descubrir y discutir problemas, en buscar soluciones a los mismos y en promover el aprendizaje común o aprendizaje mutuo; eso que nos invita a intercambiar saberes entre aquellas personas con las que convivimos. Y el enojo emerge cuando se prioriza lo administrativo, cuando hay una participación obligada en opciones de capacitación y actualización, y, además, cuando ésta se da en horarios que exigen a los docentes prolongar sus jornadas de trabajo de manera excesiva.

Por su parte Roman (2020) manifiesta que quienes participan directamente en el proceso formativo son superados por la propia tecnología en relación con el número de dispositivos que tiene, la ubicación geográfica y si tiene conectividad a internet; asimismo los socioemocionales incluyendo maneras de aprender, comunicar y en el uso de habilidades digitales.

\section{Marco teórico}

Uno de los actores esenciales de la contingencia actual es el docente quien de acuerdo a García (2020) son varias las funciones que debe desempeñar en esta modalidad, lo que implica un gran conocimiento y actualización de información actual; donde el intercambio de comunicación debe ir de acuerdo con el conocimiento pedagógico actual, por lo cual siempre debe darse énfasis a la calidad de la educación $\mathrm{y}$ en ningún momento cometer errores que provoquen situaciones confusas entre el buen trato o empatía entre docente y alumnos con ser permisivo.

En ese sentido uno de los retos que esta modalidad no escolar presenta son: que los estudiantes pueden estar viendo su celular mientras está la sesión de clases, observando la repetición de un partido del deporte de su elección por televisión por cable, desayunar, comer, chatear. Esto quiere decir que, al ubicar a la educación dentro del hogar, ambos participantes tanto estudiante como maestro se exponen a una serie de distractores por lo que se hace necesario innovar o tener herramientas que permitan disminuir esta situación para una adecuada docencia desde casa.

Dentro de este panorama es necesario definir y entender el rol del docente; por lo tanto para Brandt (2018; citado en García, 2020) un docente es aquel que se identifica con los estudiantes, es decir se ubican en su rol; saben utilizar el reconocimiento cuando se requiere, son sumamente responsables, seguros en sus habilidades con deseos de crecer, respetan la dignidad y valor de cada persona, aceptan a los alumnos como realmente son, son justos y objetivos comparados con otras personas, les gusta cambiar o experimentar cosas nuevas, nunca se precipitan en tomar conclusiones precipitadas, son pacientes $y$ saben potenciar las habilidades de cada uno de sus estudiantes. Esta conceptualización se refiere básicamente a habilidades y características que el docente debe tener, pero además falta el compromiso por parte de los estudiantes y que se cuente con los requerimientos y escenarios necesario por parte también ellos para que se cumplan los propósitos de su formación. 
También se sabe que habrá una serie de distractores que se presentan y es ahí donde debe surgir la innovación y creatividad por parte del docente; aunado a que debemos recordar que la tecnología en muchas ocasiones falla sobre todo cuando no se cuenta con una buena conectividad.

Por otra parte Nieto (2012) refiere que la educación virtual se ha perfilado como un remedio ante los retos actuales, ofreciendo amplias oportunidades de acceder a la educación a los grupos sociales que han estado desatendidos, como a otros grupos que por necesidades de ubicación así lo necesitan, ofreciendo y garantizando calidad de los servicios educativos que se prestan, utilizando toda la tecnología al alcance de los procesos educativos y promoviendo que se innove alrededor de lo que la Educación requiere.

Sin embargo, no debemos olvidar la contraparte que son las limitaciones o problemas a los cuales se enfrentan los estudiantes o en su caso el docente, cuando no cuenta con las herramientas necesarias para que este proceso fluya adecuadamente y se logre el objetivo de lograr una educación con calidad.

Britez (2020) dice que con la suspensión de las clases de manera presencial la virtualidad pasó a ocupar un lugar muy importante en la vida tanto del docente como en la familia de los estudiantes. En la mayoría de las instituciones educativas se empezaron a utilizar plataformas digitales y se empezó a apoyar en diferentes recursos tecnológicos como whapsapp, zoom, videoconferencias, aun cuando no todos los docentes están familiarizados con su uso, pero la realidad es que no tuvieron opción.

Otro problema que se ha presentado son los padres que tienen que acompañar en las tareas a sus hijos y no tienen la preparación adecuada, desconocen el uso de ambientes virtuales, se les hace difícil el uso del celular, el costo de la conectividad. En ambientes universitarios las carreras que demandan actividades que no pueden realizarse de manera virtual se han visto afectadas severamente, aunado a un gran número de dificultades que suceden a cada momento, todos los días, en muchos casos.
La pandemia solo hizo visible lo que ya sabíamos, las precarias condiciones de conectividad y acceso a este tipo de modalidad, aun cuando el uso de los teléfonos inteligentes es algo común, el manejo de la tecnología para fines educativos es algo totalmente diferentes. Están surgiendo aspectos y situaciones que no habíamos tomado en cuenta y para los cuales no estamos preparados.

Por otra parte, Ferrer (2020) en relación a la pandemia nos dice que es importante también saber cómo reaccionan los seres humanos ante tanta información relacionada con el tema que ha impactado al mundo. Debemos buscarle el lado positivo a la situación actual que nos ha tocado vivir, darnos cuenta de las buenas acciones de la gente, la solidaridad y apoyo que se ha mostrado, las diferentes acciones hacia nuestras familias y sociedad en general.

En este contexto debemos prestar atención al aspecto psicológico de los docentes, estudiantes y población en general. Muchos de ellos han estado en situación de pánico, estrés, ansiedad, derivado del miedo al contagio o ante tanta información del tema pues el tema de la pandemia invade noticieros, redes sociales, pláticas entre amigos, familia, negocios.

Actualmente muchos docentes padecen enfermedades psicológicas por el miedo o temor que representa el virus y debemos prestarle atención a estos y otros factores que en determinado momento pueden afectar a los estudiantes pues un docente con problemas como puede transmitir adecuadamente a sus estudiantes sus conocimientos.

No debemos olvidar las limitaciones tecnológicas de los docentes, el conocimiento en el manejo de las tecnologías de información, plataformas digitales, redes sociales, etc. Es en este panorama donde han surgido muchas dificultades de los docentes porque recordemos que muchos de ellos no son tan jóvenes y no cuentan con las habilidades y destrezas que hoy en día la mayoría de los jóvenes y adolescentes tienen. Coloquialmente decimos nacieron con una computadora y celular, es decir en la era de la tecnología. 
Martínez \& Virto (2020), refiere que la actual crisis sanitaria por la que está atravesando el planeta y las consecuencias que trae consigo han puesto la atención en las desigualdades educativas. Desde que empezó la pandemia $y$ sobre todo el resguardo o confinamiento ha sido tema de interés y debate como mantener y acompañar el trabajo de la educación desde el hogar. Las opiniones vertían en si mantener o terminar contenidos $\mathrm{o}$ aprovechar los espacios de tiempo para otro tipo de aprendizaje.

De cualquier modo, la respuesta fue diferente en cada país, estado, población, comunidad, región e inclusive entre las instituciones educativas ya que las condiciones son diferentes. Incluso los docentes que laboran en una ciudad son diferentes a los que trabajan en pueblos o comunidades rurales, los primeros tienen acceso a los recursos tecnológicos y herramientas computacionales mientras que los segundos se enfrentan a escenarios de desigualdad y donde la mayoría de sus estudiantes y docentes carecen de accesibilidad y conectividad.

En este escenario la educación pública universitaria juega un papel primordial en nuestra sociedad, además de un espacio donde los individuos se forman, y que coadyuva a una organización social más completa quien será el principal eje económico de muchas generaciones presentes y futuras. Asimismo, es un lugar en el cual se puede dejar de lado las desigualdades sociales, formando personas con valores como igualdad, convivencia, justicia, sobre todo en estos momentos donde las condiciones desigualdad e injusticia son más visibles.

El objetivo general de la investigación es identificar y analizar los principales retos educativos a los cuales se enfrentan los docentes de la Unidad Académica del Norte del Estado de Nayarit durante la pandemia de COVID-19.

\section{Entre los objetivos específicos son:}

Identificar los problemas educativos $\mathrm{y}$ tecnológicos que enfrentan los docentes de la UANEN.

Analizar los principales problemas que se detectaron en la investigación.
Proponer algunas estrategias a la UANEN para beneficiar a los docentes en tiempos de emergencia sanitaria.

El tipo de investigación es transversal y cuantitativa ya que muestran datos obtenidos por una ocasión y en un espacio de tiempo determinado. Asimismo, es una investigación de campo por la forma y naturaleza en que se obtuvo la información. Para llevarla a cabo se utilizó un cuestionario reconocido y aplicado por la Universidad Nacional Autónoma de México (2020) y el cual ha sido adaptado. La población encuestada fue de 16 docentes que participan en los 5 programas educativos que se atienden en la Unidad Académica (Contaduría, Administración, Mercadotecnia, Ciencias de la Educación y Derecho), el instrumento fue implementado a través de un formulario de google enviado por correo electrónico.

De la totalidad de los académicos encuestada el $56.3 \%$ son del sexo femenino y el $43.8 \%$ masculinos, por lo que en la Unidad Académica el personal femenino predomina en relación con el masculino (véase el gráfico 1).

En relación con el rango de edad, el 50 $\%$ de los docentes se encuentra entre las edades de 41 a 50; el $43.8 \%$ tienen de 31 a 40 años y; el resto de 51 a 60 (véase el gráfico 2). Este dato es importante en lo que se refiere al conocimiento y habilidades en el uso de redes sociales, tecnología, plataformas, ya que los jóvenes han podido estar en contacto con la era digital con mucha más anticipación que los docentes maduros.

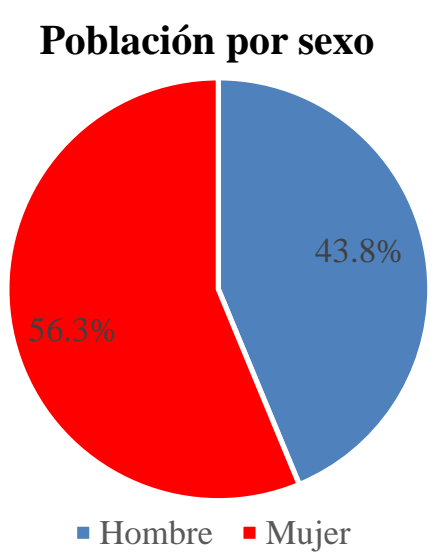

Gráfico 1 Población por sexo 


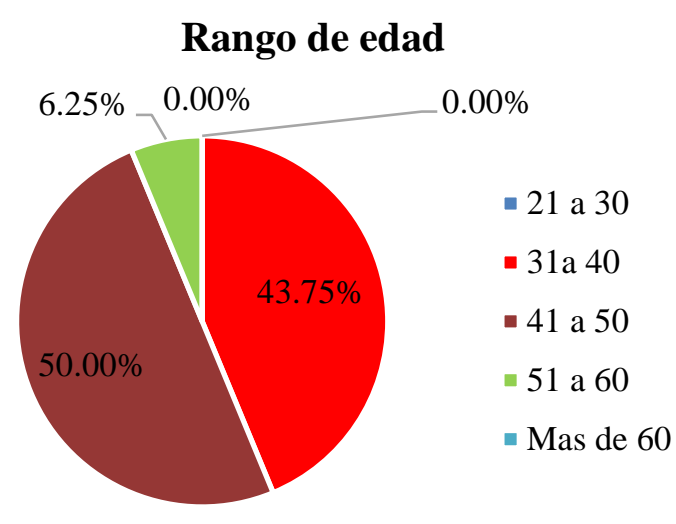

Gráfico 2 Rango de edad del personal docente de la Unidad Académica

\section{Tipo de Contrato}

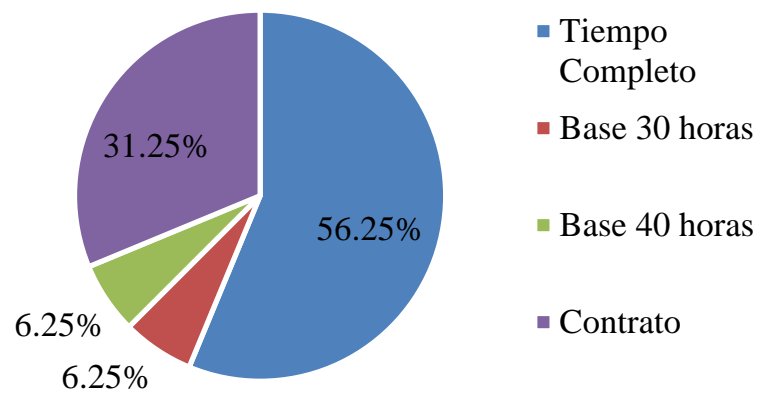

Gráfico 3 Tipo de contratación

De acuerdo con el tipo de contratación el $56.3 \%$ de los docentes son de base de tiempo completo, el $31.3 \%$ son de contrato, mientras que de base con 20 y 40 horas son el $6.3 \%$ respectivamente.

\section{Número de grupos por periodo}

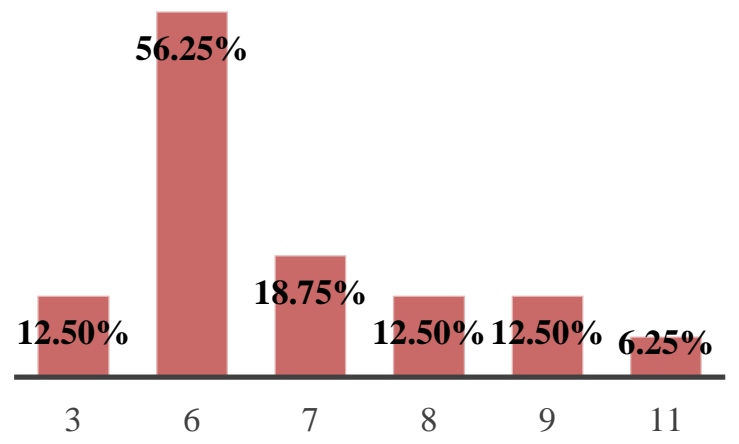

Gráfico 4 Número de grupos en el periodo agostodiciembre de 2020
El número de grupos que se atienden por docente va desde seis, siendo éste el máximo a trabajar y como mínimo tres. En la medida en que la atención a estudiantes crece en número obviamente los problemas pueden incrementarse o en su caso potenciarse.

\section{Estudiantes en el semestre agosto- diciembre}

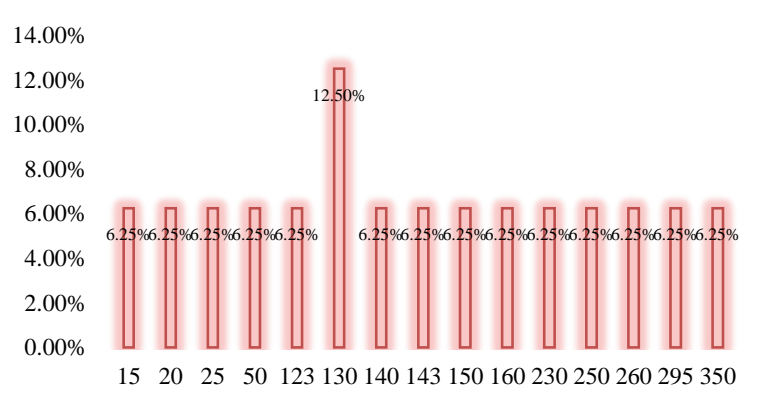

Gráfico 5 Población estudiantil de la Unidad Académica

Se atiende una población estudiantil de $350,260,230,150,140,123$ respectivamente mientras que otros atienden 15 o 25 alumnos. Este desequilibrio afecta directamente a los docentes con mayor número de estudiantes pues obviamente nunca será igual la atención de 15 estudiantes a un número mayor. Por lógica un número mayor implica mayor atención, tiempo para revisar, retroalimentar, atender dudas y dar seguimiento a su proceso de aprendizaje.

\section{Apoyo institucional que has recibido de la Universidad}

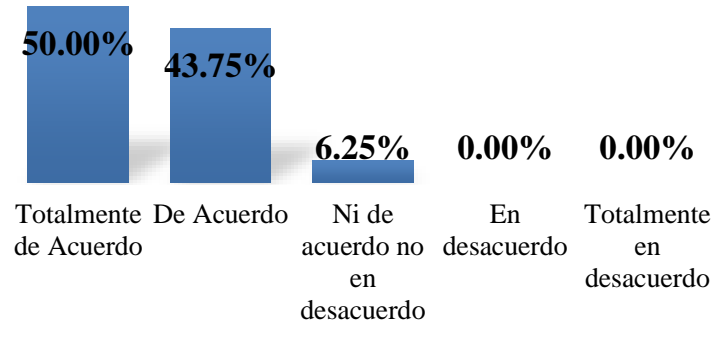

Gráfico 6 Apoyo institucional

En el aspecto del apoyo que brinda la UAN para las actividades a distancia, el $43 \%$ de los docentes encuestados manifestaron que, si recibieron el apoyo institucional, por otro lado, el $43 \%$ dijo no estar de acuerdo de haber recibido el apoyo necesario para llevar a cabo las actuales actividades, mientras que el $2 \%$ reiteró no estar ni de acuerdo ni en desacuerdo y el $2 \%$ respondió que está totalmente en desacuerdo, con los apoyos recibidos. 


\section{Problemáticas tecnológicas}

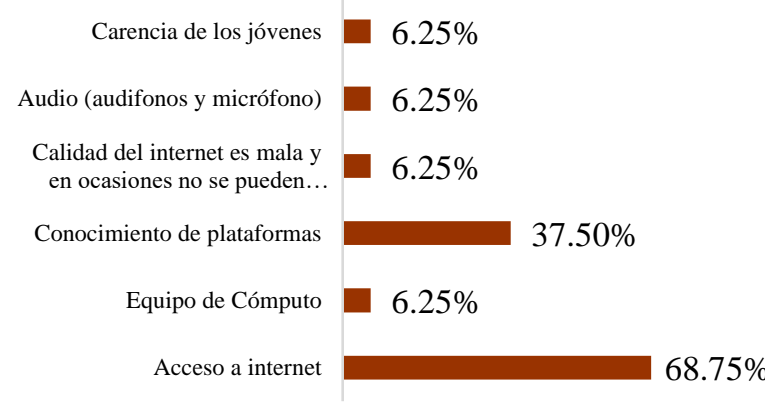

$0.00 \% 20.00 \% 40.00 \% 60.00 \% 80.00 \%$
Gráfico 7 Problemáticas desde el aspecto tecnológico

Respecto a las problemáticas tecnológicas que el docente ha presentado el $68.8 \%$ manifestó que el acceso a internet es el más frecuente, sobre todo por la región geográfica en donde se ubica la Unidad Académica; el $37.5 \%$ refirió sobre el conocimiento en el uso de las plataformas educativas; el $12.5 \%$ el no contar con equipo de cómputo; el $6.3 \%$ carencias económicas de los jóvenes y de igual forma el $6.3 \%$ respondió que la calidad del internet es muy mala y el audio respectivamente.

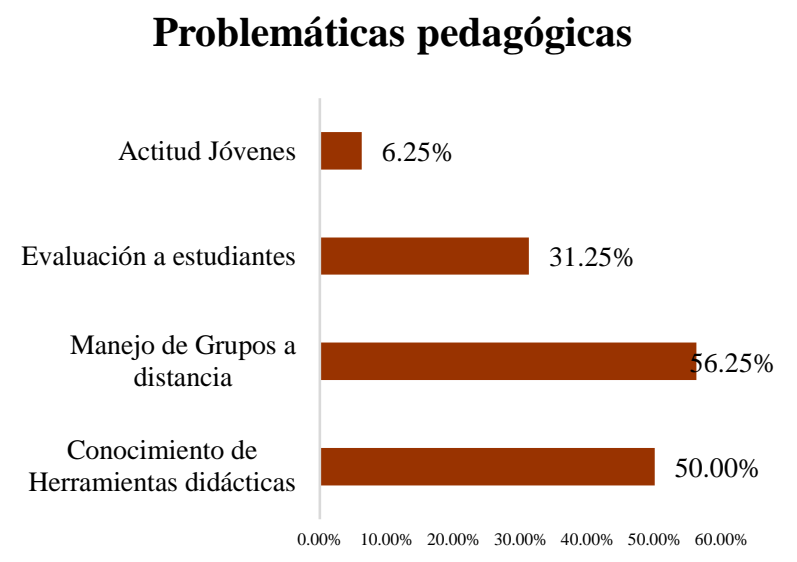

Gráfico 8 Problemáticas desde el aspecto pedagógico

Las principales problemáticas pedagógicas que enfrentan los docentes son el manejo de grupos a distancia $(56.3 \%)$, seguido del desconocimiento de herramientas didácticas (50\%); en el aspecto de cómo evaluar a los estudiantes el $31.3 \%$ también se identificó como una problemática, por último, la actitud de los jóvenes al estar trabajando en esta modalidad también fue considerada como parte de esta categoría (6.3\%).

\section{Problemáticas de lógistica}

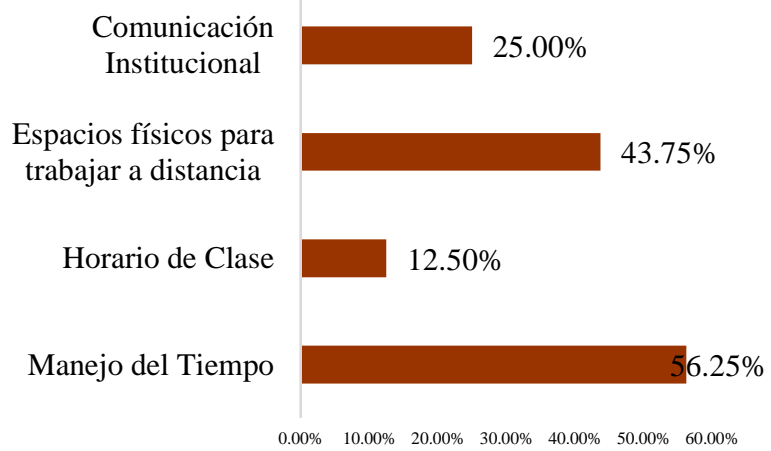

Gráfico 9 Problemáticas de logística

En relación con la pregunta sobre los problemas de logística, el de mayor incidencia es el manejo del tiempo, el cual refiere a la sobresaturación de actividades que se tuvieron que realizar para el cambio en la modalidad de trabajo (56.3\%); el segundo son los espacios físicos para poder llevar a cabo las actividades $(43.8 \%)$ y por ultimo las estrategias para comunicarse institucionalmente $(25 \%)$ y por último los horarios de clases (12.5).

\section{Problemáticas socioafectivas}

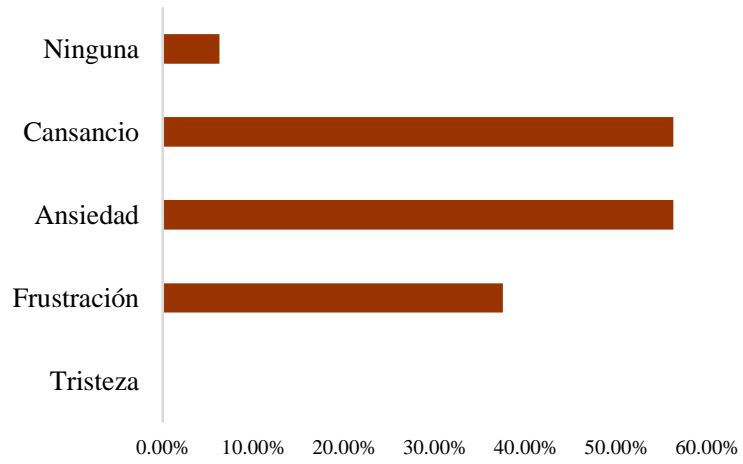

Gráfico 10 Problemáticas socioafectivas

Otro tipo de problemática a la que se enfrentan los docentes tienen que ver con la parte socio afectiva y como ésta se ha visto afectada por todos estos cambios, entre ellos el cansancio mental y físico, así como la ansiedad, ambos con un $56.3 \%$; la frustración representa el $37.5 \%$ y solamente el $6.3 \%$ respondió no haber presentado ningún problema durante estos cambios. 


\section{Problemáticas tecnológicos de los} estudiantes

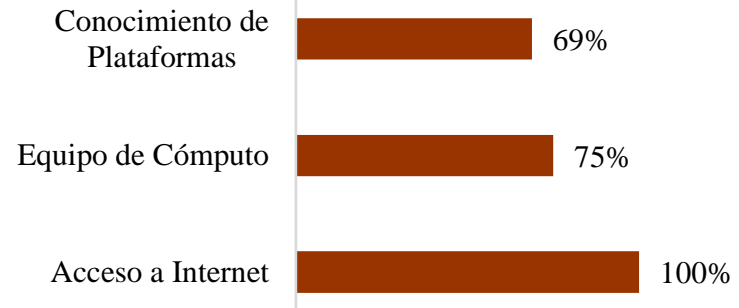

$0 \% \quad 20 \% \quad 40 \% \quad 60 \% \quad 80 \% 100 \% 120 \%$

Gráfico 11 Problemáticas tecnológicas de los estudiantes desde la percepción de los docentes

Esta pregunta se plantea desde la perspectiva de los docentes y sus vivencias con los estudiantes, pues uno de los principales problemas a los que éstos se enfrentan es la conectividad debido a su lugar de origen, muchos de ellos vienen desde comunidades donde el acceso a internet es precario, no hay buena señal e incluso todavía compran tarjetas telefónicas para poder acceder a las actividades. También es importante destacar que no cuentan con un equipo de cómputo adecuado, y no conocen el funcionamiento de las plataformas educativas (véase el gráfico 11).

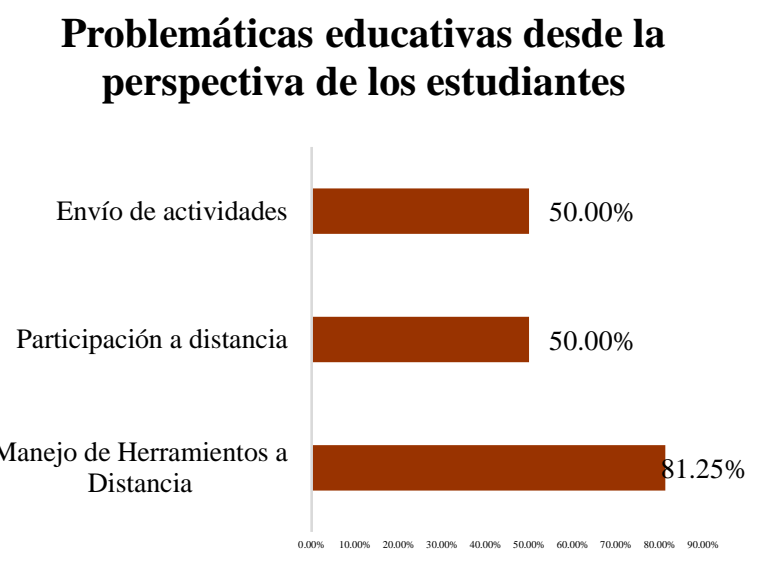

Gráfico 12 Problemáticas educativas de los estudiantes desde la percepción de los docentes

Los principales problemas educativos identificados son en el manejo de herramientas de educación a distancia, seguido de la participación y envío de actividades respectivamente (véase gráfico 12 ).

\section{Problemáticas de logistica}

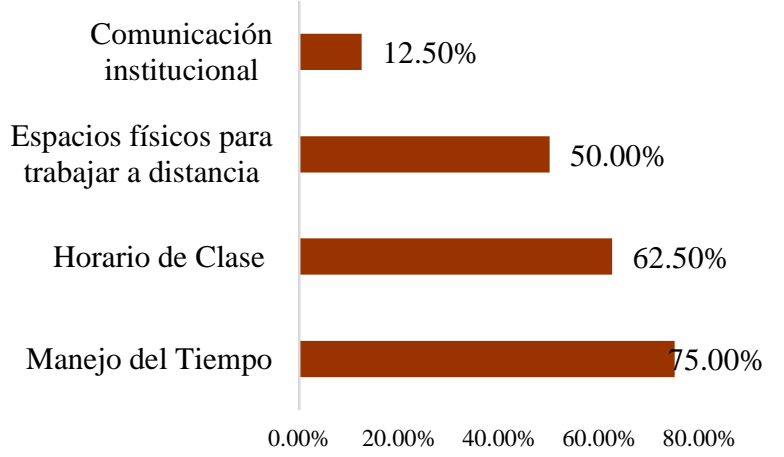

Gráfico 13 Problemáticas de logística de los estudiantes desde la percepción de los docentes

Las principales problemáticas de carácter logístico que los estudiantes desde la percepción de los docentes están relacionados con el manejo del tiempo con un $75 \%$, los horarios de clases con $56.3 \%$ y espacios físicos para trabajar a distancia respectivamente. La comunicación institucional resultó con un 12.5 $\%$.

\section{Problemáticas socioafectivas}

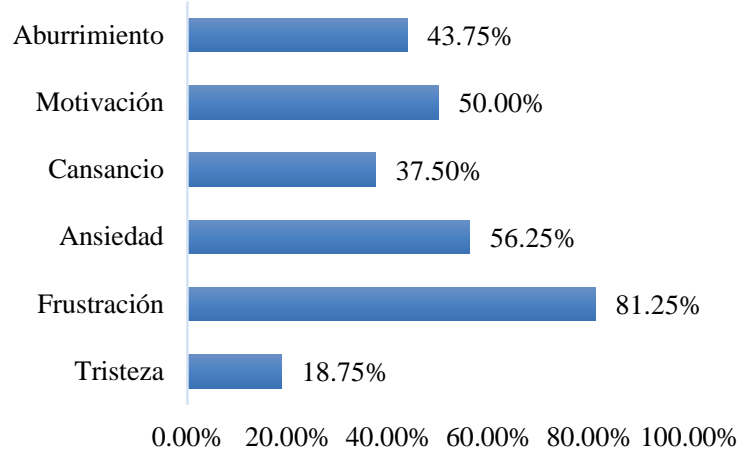

Gráfico 14 Problemáticas socioafectivas de los estudiantes

Las problemáticas socioafectivas a las que se están enfrentando los estudiantes son la frustración, seguida de la ansiedad, motivación, cansancio, aburrimiento, derivado de la situación actual el cual es compatible con los que enfrentan los docentes (véase el gráfico 14). 


\section{Recursos tecnológicos}

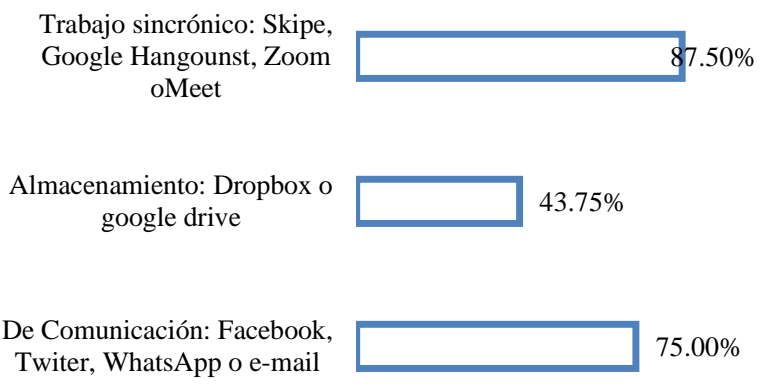

Gráfico 15 Recursos tecnológicos utilizados para las actividades

En relación con los recursos tecnológicos que los docentes utilizan para sus actividades a distancia el $100 \%$ respondió que usa recursos de trabajo académico como Moodle, Google Suite, Google Classroom, Teams; el $87.5 \%$ recursos de trabajo sincrónico como Skype o Hangouts; el $75 \%$ recursos de comunicación como Facebook, Twitter, WhatsApp y $43.8 \%$ utiliza recursos de almacenamiento como Dropbox, drive, entre otros.

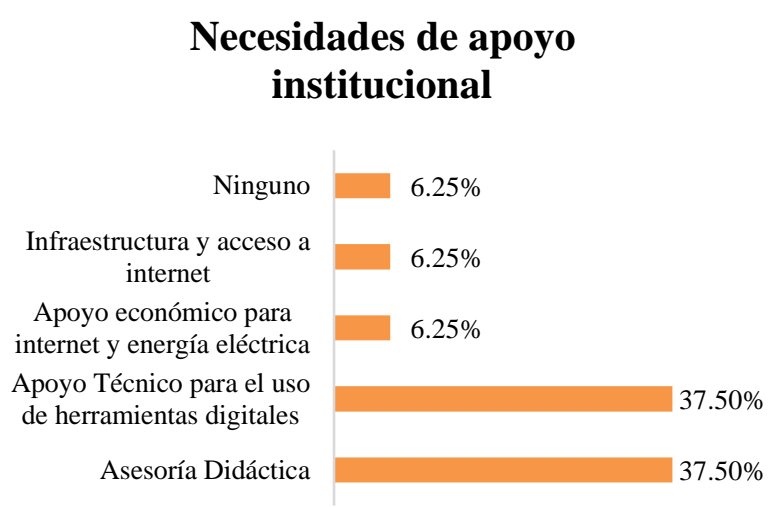

$0.00 \% 10.00 \% 20.00 \% 30.00 \% 40.00 \%$

Gráfico 16 Necesidades de apoyo institucional

Los docentes manifiestan que requieren el apoyo técnico para el uso de las herramientas tecnológicas $(56.3 \%)$, el $37.5 \%$ manifiestan necesitar asesoría didáctica, el $6.3 \%$ apoyo económico para el pago de internet, al igual que infraestructura y acceso a internet.

\section{Medios de información}

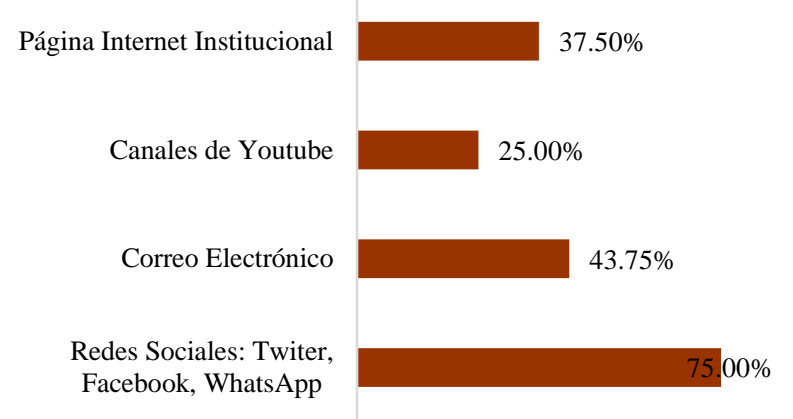

Gráfico 17 Medios de información

Analizando los resultados obtenidos se identifica que los medios por los cuales los docentes les gustaría recibir más información referente a cursos de capacitación o actualización son en primer lugar las redes sociales con un $75 \%$, seguido del correo electrónico con un $43.8 \%$, páginas de internet institucionales con un $37.5 \%$ y canales de YouTube un $25 \%$.

Además de estos resultados, los participantes realizaron una serie de propuestas que, desde su perspectiva se deben de desarrollar a nivel institucional en apoyo a la docencia durante la contingencia e incluso futuras emergencias sanitarias, las cuales se enlistan a continuación:

- Apoyar a los maestros económicamente y que sean flexibles con la exigencia de diseños instruccionales de las unidades de aprendizaje.

- Incrementar en la cobertura para proporcionar herramientas de cómputo a los docentes y estudiantes.

- $\quad$ Fortalecer la línea de soporte técnico.

- Solucionar los problemas administrativos (listas de estudiantes en tiempo).

- Brindar capacitación pedagógica para manejo a distancia y conectividad a internet ya que es ineficiente. Ademas que este proceso sea de forma secuencial y no tan apresurada.

- Incrementar el a internet en la universidad para los estudiantes que no tienen en su casa. 


\section{Conclusiones y recomendaciones}

De acuerdo con los resultados obtenidos es importante destacar que la mayoría de los docentes coinciden en que parte de las problemáticas más frecuentes que han tenido son acceso a internet y el conocimiento de plataformas educativas. En relación con la logística el manejo y organización del tiempo y el no contar con espacios adecuados para llevar las actividades, han incido en problemas como el cansancio físico y el incremento de la ansiedad.

Por otra parte, los problemas educativos más frecuentes son el manejo de herramientas de educación a distancia por parte de docentes y alumnos. En este contexto los docentes manifestaron que el apoyo institucional que requieren para realizar adecuadamente su trabajo es el apoyo técnico para el uso de herramientas tecnológicas.

Es importante mencionar que este trabajo de investigación se realizó a inicios del periodo escolar en el mes de septiembre, por lo que a la distancia se ha podido observar que la UAN ha brindado apoyo tanto a docentes como estudiantes a través de convocatorias para incrementar la conectividad y accesibilidad tanto de docentes como estudiantes, por ello se han otorgado tarjetas con internet gratuito con el objetivo de facilitar el proceso educativo. En la misma vertiente ha contribuido en la formación de los docentes que laboran en la institución brindándoles acceso a cursos relacionados con el uso adecuado de plataformas educativas.

Es de reconocer los esfuerzos que la universidad ha hecho en ese sentido pues la emergencia sanitaria trajo un mundo de desconocimiento para todos los actores y vino a cambiar la forma y estilo de vida. Ante este panorama las acciones se deben continuar y mejorar la capacitación a los docentes en el uso de plataformas educativas y recursos tecnológicos. También se ha de prestar atención a la salud emocional de los docentes que realmente se ha visto afectada, atender los problemas de ansiedad, a través de talleres de autoayuda, apoyados por profesionales de la salud, pues los retos son muchos y seguirán dándose conforme se vayan dando este tipo de emergencias sanitarias.

\section{Referencias}

Britez, M. (2020). La educación ante el avance del COVID-19 en Paraguay. Comparativo con países de la Triple Frontera. 1-13.

García, M. D. (2020). La docencia desde el hogar. Una alternativa necesaria en tiempos del Covid 19. POLO DEL CONOCIMIENTO, p. 304-324. (Edición núm. 44) Vol. 5, No 04 recuperado de http://polodelconocimiento.com/ojs/index.php/e $\mathrm{s}$

Lucía Martínez Virto, A. A. (2020). Escuela Post-Covid: La Oportunidad de Repensar la Educación en Clave Inclusiva. Revista Internacional de Educación para la Justicia Social, 1-7.

Melchor Sánchez Mendiola, Ana M. del Pilar Martínez Hernández, Ruth Torres Carrasco, Mercedes de Agüero Servín, Alan K. Hernández Romo, Mario A. Benavides Lara, Carlos A. Jaimes Vergara, Victor J. Rendón Cazales. (2020). Retos educativos durante la pandemia de Covid 19, una encuesta a profesores de la UNAM. Revista Digital Universitaria Ahead of Print 2020, 1-23.

Nieto, Rafael. A. (2012). Educación virtual o virtualidad de la Educación. Revista Historia de la Educación Latinoamericana, 137-150. vol. 14, núm. 19, julio-diciembre recuperado de http://www.redalyc.org/articulo.oa? $\mathrm{id}=8692697$ 6007

Piña, F. (2020). El COVID 19: Impacto psicológico en los seres humanos. Revista Arbitrada Interdisciplinaria de Ciencias de la Salud. SALUD Y VIDA, 188-199. Volumen 4. Número 7. Año 4. Enero - Junio 2020 recuperado

http://dx.doi.org/10.35381/s.v.v4i7.670

Román, José A. (2020).La educación superior en tiempos de pandemia: una visión desde dentro del proceso formativo, Revista Latinoamericana de Estudios Educativos (México), vol. L, núm. Esp.-, 2020 recuperado de

https://www.redalyc.org/jatsRepo/270/2706323 7017/html/index.html 
Maryinez-Gomez, German I. (2020). Los docentes de educación básica en México ante el COVID-19 ¿La emergencia como principio de innovación docente?, reuperado de http://www.comie.org.mx/v5/sitio/2020/05/26/1 os-docentes-de-educacion-basica-en-mexicoante-el-covid-19-la-emergencia-comoprincipio-de-innovacion-docente/. 